\title{
Dossier Le naturel et la qualité Équiper des filières durables ? L'élevage bio en Belgique
}

\author{
Pierre Stassart ${ }^{\mathrm{a}}$, Daniel Jamar ${ }^{\mathrm{b}}$ \\ a Sociologue, SEED, Université de Liège (ULg), Avenue de Longwy 185, 6700 Arlon, Belgique \\ b Agronome, Section Systèmes agraires, Centre wallon de recherches agronomiques (CRA-W), Rue du Serpont 100, \\ 6800 Libramont, Belgique
}

L'organisation d'une filière biologique ne va pas de soi. Au sein du dossier proposé par Natures Sciences Sociétés, ce forum permet d'illustrer la complexité sociale et technique de la construction d'une filière qui pose des problèmes que seule une démarche d'esprit interdisciplinaire, de la part des acteurs eux-mêmes, peut traiter. Ils le font en mobilisant des normes, des connaissances et des images, soit un effort de construction qui rappelle que les référentiels d'un développement durable supposent une constante et difficile mise à l'épreuve.

La Rédaction

\section{Mots-clés :}

développement

durable ;

filière ;

élevage ;

sociologie;

zootechnie ;

recherche-intervention

\section{Keywords:}

sustainable development; agrifood chains; livestock breeding; sociology; animal husbandry; intervention research
Résumé - L'agriculture biologique est actuellement à un tournant de son développement : sollicitée de plus en plus fréquemment pour entrer dans une filière de type long, elle apparaît comme "sous-équipée », se confrontant ainsi d'autant plus durement à une filière conventionnelle qui, elle, est "suréquipée ". Les auteurs analysent ce déplacement difficile en se référant au débat anglo-saxon sur la «conventionnalisation ». Ensuite, plutôt que de l'analyser en termes de "conversion de système de production ", ils proposent la notion de « référentiel » qui envisage et compare l'équipement des deux filières en termes de normes, de connaissances et d'images. Cette grille de lecture permet à la fois de comprendre le caractère non questionné et non questionnable de la filière conventionnelle et de mettre en évidence la fragilité de la filière bio à se maintenir comme alternative durable.

\begin{abstract}
Outfitting sustainable production lines: organic livestock in Belgium. Organic agriculture is at a turning point in its development: it is increasingly being pressed to enter extended agrifood chains involving supermarket chains and appears "under-equipped" for this venture as opposed to the "overequipped" conventional agrifood chains. The authors analyze this difficult conversion by referring to the debate on "conventionalisation" of organic production. Rather than analyzing it in terms of "production system conversion", they propose the notion of "referential", which considers and compares the equipment resources in both agrifood chains in terms of norms, knowledge and images. These resources are the means by which the agrifood chains create a shared understanding of the expected product by coherently articulating the heterogeneous actors involved from the production through to the consumption end. This analysis leads to understanding the unquestioned and unquestionable character of the conventional agrifood sector. It also highlights the difficulty of building a sustainable reference base that is meant to be distinct from conventional agrifood reference systems, but has no other resources than to follow these already established systems. The fragility of the organic industry in maintaining itself as a sustainable alternative raises the issue of the "equipment" of the conventional agrifood sector for engaging in a sustainable development logic.
\end{abstract}

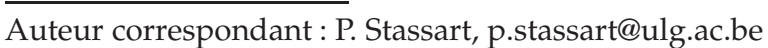


Si l'agriculture biologique a aujourd'hui pignon sur rue et fait débat, c'est parce que cette forme particulière de production agricole a acquis, au cours des quinze dernières années, une forme de reconnaissance qui en fait maintenant une alternative reconnue aux modes conventionnels de production et de consommation. Cette reconnaissance, d'abord institutionnelle et sociétale, s'est vue validée dans un second temps par le marché. En effet, dans le sillon des crises alimentaires de la fin des années nonante, le bio est sorti des réseaux spécialisés où il fut longtemps confiné, pour s'imposer comme gamme de plus en plus large dans les linéaires de la grande distribution.

Les conséquences de l'extension du marché bio par l'allongement des filières représentent une mise à l'épreuve et font débat. Ce débat, axé sur le thème de la «conventionnalisation » de l'agriculture biologique, divise les auteurs anglo-saxons. Buck et al. (1997), s'inscrivant dans la tradition de l'économie politique, ont décrit, à partir du cas californien, des processus d'appropriation par l'industrie d'aval. Smith et Marsden (2004) montrent comment les grands groupes de distribution sont en train de se réapproprier la rente du bio aux dépens des producteurs par un mécanisme classique de "squeeze price» (croissance des coûts de production et baisse des prix de vente). Coombes et Campbell (1998), de même que Hall et Mogyorody (2001), critiquent cette position en appuyant leur argumentation sur la coexistence et les possibles synergies entre «bio-industrie » et producteurs s'identifiant au «mouvement historique » du bio.

Pour contribuer à ce débat sur la conventionnalisation, le contexte belge et les crises qu'a connues le secteur conventionnel ces dernières années nous offraient une situation inédite : l'entrée massive des industriels et des distributeurs sur le marché de la viande bio. Cette rupture avec les réseaux de proximité spécialisés (magasins diététiques, vente directe) a amené la production de viande bovine bio à une confrontation directe avec un référentiel national conventionnel extrêmement cohérent, dont le Blanc-Bleu Belge culard est le porte-parole. Nous avions là un terrain qui pouvait nous permettre de comprendre, dans un premier temps, comment une forte référence conventionnelle interagissait avec le contenu de la conversion au bio et, dans un second temps, à quelles conditions la conversion au bio pouvait déployer toutes ses potentialités. Cette question a été abordée à travers une recherche-intervention (Hubert, 2002), dans le cadre d'une convention de collaboration entre distribution et producteurs bio qui nous a permis de tester notre hypothèse ${ }^{1}$.

\footnotetext{
${ }^{1}$ Cet article s'appuie sur les résultats du projet de recherche "Comment l'agriculture biologique contribue-t-elle aux modes de production et de consommation durables? ", contrat CP 19/191, Programme d'appui au développement durable,
}

Le problème posé est celui d'éleveurs de bovins qui se convertissent au bio en s'engageant dans une démarche de filière longue. Pour ce faire, ils doivent en effet se construire de nouveaux points de repère (bio), tout en s'appuyant sur ce qu'ils connaissent : la référence conventionnelle omniprésente, celle du Blanc-Bleu Belge. Sur le terrain, deux cas de figure sont observés. Certains éleveurs partent de ce qu'ils connaissent : ils conservent l'équipement conventionnel qu'ils adaptent pas à pas aux contraintes du cahier des charges bio. C'est ainsi qu'obligés de changer de race pour éviter la césarienne, ils s'orientent vers deux races dont la conduite peut devenir fort proche de celle du Blanc-Bleu Belge : la Blonde $\mathrm{d}$ 'Aquitaine et la Limousine. Nous verrons cependant comment apparaissent des tensions, des fissures derrière la belle unanimité que crée la certification. D'autres éleveurs font le choix radical de s'aventurer en dehors des références existantes : ils se convertissent à des races rustiques telles que la Salers ou l'Angus et/ou expérimentent en circuit court. Mais, après plusieurs années de tâtonnements infructueux, nombreux sont ceux qui renoncent : le bétail que l'on a cherché à commercialiser comme gras et bio rejoint maintenant les filières du maigre conventionnel ${ }^{2}$. Enfin, certains, à travers une opération d'hybridation, amorcent un mouvement de retour vers le conventionnel en croisant le troupeau de Salers avec un taureau Blanc-Bleu Belge pour produire des bêtes alliant conformation cularde de l'individu et caractère rustique $\mathrm{du}$ troupeau de vaches allaitantes. Notre hypothèse est que le problème que pose dans ce cas la conversion, et donc la construction d'une nouvelle référence de filière par rapport à un cahier des charges donné, est celui du passage d'une référence «suréquipée » (conventionnelle) à une référence "sous-équipée » (bio). Ce décalage pousse les éleveurs bio à rester aussi proches que possible du modèle existant pour bénéficier de ses ressources, mais cette tentative d'hybridation, favorisée par l'opacité de ce modèle, inhibe leur potentiel d'innovation.

Dans une première partie, nous aborderons de façon détaillée la notion de référentiel de filière en l'appliquant à la filière bovine conventionnelle qui s'appuie, notamment, sur la race Blanc-Bleu Belge. Dans une seconde partie, nous montrerons comment le référentiel bio se construit. Et enfin, dans la conclusion, nous réintégrerons la question de la durabilité de la production biologique, non pas en partant de l'impact environnemental des systèmes de production, globalement reconnu (Lampkin et Padel, 1994), mais en interrogeant la durabilité de l'ensemble de la filière et les conséquences de l'interdépendance entre ses différents maillons.

\footnotetext{
Service public fédéral de programmation politique scientifique (Stassart et al., à paraître).

${ }^{2}$ Environ $75 \%$ de l'élevage bovin bio viandeux est écoulé
} vers le marché conventionnel de l'engraissement. 


\section{Cohérence de filière : la notion de référentiel}

Un diagnostic partiel de la filière bio, réalisé dans un premier temps de notre recherche, nous a permis d'identifier quatre points de tension entre le respect du cahier des charges bio et les pratiques observées dans les systèmes d'élevage et d'engraissement. Ces points de tension concernent : (i) le respect de l'obligation de pâturage ; (ii) le pourcentage d'aliments bio dans l'alimentation des bovins, en particulier pour les taureaux confinés en étable durant la phase d'engraissement-finition; (iii) le type de traitement allopathique préventif ou curatif du parasitisme (vers pulmonaire et gastro-intestinaux), dont la pression s'accroît fortement avec l'obligation de pâturage; (iv) le décalage de trois ans entre la période de reconversion au bio ( 2 ans) et la période de changement de race ( 5 ans) en raison de l'interdiction de la césarienne systématique qu'impose la race d'origine, le Blanc-Bleu Belge culard. Ces quatre points de tension - pâturage, alimentation, prévention et choix de la race -, sources de contradiction entre pratiques et respect du cahier des charges, touchent à la fois à l'esprit et à la lettre du règlement bio. Ils révèlent les difficultés de la traduction de la norme bio, difficultés dont l'exploration est au cœur de notre interrogation.

\section{Comment interpréter les tensions liées à la traduction nationale du référentiel bio?}

Passant rapidement sur deux clés d'interprétation que certains pourraient proposer, mais que nous avons invalidées, nous allons en développer une troisième qui constitue l'hypothèse centrale de ce papier. La première clé d'interprétation est juridico-administrative : les tensions observées appelleraient un ajustement entre règle et contrôle. Deux issues sont alors possibles : les pragmatiques suggéreraient que les règles du cahier des charges soient adaptées aux exigences du marché et qu'ainsi, les normes bio s'ajustent à cette naturalisation de la réalité; les dogmatiques, en revanche, objecteraient que le contrôle de la mise en œuvre des règles, trop lâche, devrait être durci pour éviter de voir se développer des stratégies de contournement, sources de concurrence déloyale. Nous avons écarté cette interprétation normative parce qu'en se focalisant sur la production, elle ne prenait pas en compte les effets de système liés à l'interdépendance avec les maillons d'aval de la filière, transformation et distribution, chargeant ainsi les éleveurs et leurs troupeaux d'une responsabilité que partagent de fait les autres acteurs de la filière.

Une seconde clé d'interprétation serait davantage stratégique et fondée sur les relations de domination : l'industrie agroalimentaire d'aval (abattoirs et distributeurs), nouvelle venue dans le secteur bio, reconfigurerait les processus de production agricole pour les réduire à un input de l'agro-industrie. C'est ce que l'économie politique décrit comme un mécanisme d'appropriation (Goodman et al., 1987; Buck et al., 1997). Nous avons effectivement observé que, du point de vue de la définition de la qualité, les exigences de l'agro-industrie en matière de valorisation des carcasses et de découpe orientent fortement le travail des éleveurs engraisseurs. Ainsi, les attentes de l'industrie d'abattage en termes de conformation et d'état d'engraissement des bovins entraînent certaines incompatibilités, notamment avec le respect de l'obligation de pâturage et les contraintes d'alimentation qu'impose le cahier des charges bio, comme nous l'avons évoqué dans les points de tension. Mais ces exigences des intermédiaires, transformateurs et distributeurs sontelles subies d'une manière impuissante par les éleveurs et les consommateurs bio? Ceux-ci ne peuvent-ils pas se soustraire à la logique d'appropriation des intermédiaires pour recadrer leurs échanges en cohérence avec l'esprit et la lettre du bio? Nos observations ont invalidé cette interprétation : les mêmes tensions apparaissent en situation de circuit court de type vente à la ferme, lorsque éleveurs et consommateurs sont en face-à-face.

Cela nous a donc amenés à formuler une hypothèse de type cognitif : les points de tension observés sont la manifestation d'incompatibilités entre deux cadres interprétatifs du monde - le cadre de référence national de la filière bovine conventionnelle, celui du «maigre et tendre » associé à la race Blanc-Bleu Belge, et un cadre émergent plus global, celui de l'agriculture biologique. Les incompatibilités entre ces deux cadres font émerger des tensions entre pratiques et respect des règles du cahier des charges bio. Cette hypothèse propose de questionner les conditions sous lesquelles les principes et les pratiques liés au cadre de référence ou référentiel de filière du Blanc-Bleu Belge peuvent se convertir au référentiel bio.

Nous avons développé la notion de référentiel de filière à partir du concept de référentiel sectoriel et global développé en sciences politiques (Jobert et Muller, 1987). Un référentiel de filière est une représentation commune que les acteurs se font de leur filière pour aboutir à un produit attendu ${ }^{3}$. Nous utilisons la notion de référentiel de filière pour souligner tout $d^{\prime}$ abord $q u$ 'au sein $d^{\prime}$ 'une filière composée de différents maillons ou groupes d'acteurs hétérogènes (éleveurs, engraisseurs, abatteurs, transformateurs, distributeurs, consommateurs), ceux-ci se coordonnent en fonction d'un état souhaitable qui leur permet de partager une interprétation commune du monde. Ceci ne gomme pas la diversité des pratiques, mais nous

\footnotetext{
3 Cette notion se distingue de celle d'un référentiel technique, utilisé en sciences agronomiques pour décrire un ensemble de données de références mises au point, dans un objectif de production, sur la base d'expérimentations dans des situations définies. Il constitue en fait une traduction du référentiel de filière au niveau des pratiques agricoles (de Bonneval, 1993).
} 
indique qu'il y a un modèle de filière qui agit comme cadre interprétatif et référence. Nous allons montrer comment ce cadre est à la fois partagé, implicite et articulé autour de normes, de savoirs et d'images.

\section{Le référentiel conventionnel du maigre et du tendre}

Le caractère partagé du référentiel est la traduction (Callon, 1986) que la filière effectue à partir du "produit souhaité » et qui permet d'aligner l'ensemble des acteurs sur un principe commun d'action et de maintenir, malgré son hétérogénéité, l'intégrité de la filière. $C^{\prime}$ est ainsi qu'un standard de qualité spécifique au marché de viande bovine belge s'est constitué comme cadre normatif, pour orienter l'action de la filière conventionnelle à partir du début des années septante. Ce cadre est dessiné par deux critères simples : le maigre et le tendre, double qualité de la viande que propose la filière conventionnelle belge, à l'opposé d'autres pays, tels les anglo-saxons, qui préfèrent le gras au maigre. Pour les acteurs de l'élevage, cette norme est traduite au niveau du herd-book Blanc-Bleu Belge dans l'orientation hyperviandeuse, dite «cularde», et, en aval de la filière, dans la description de la découpe belge dite « découpe anatomique » du manuel des écoles de la Fédération nationale des bouchers belges ${ }^{4}$ (Stassart, 2003a). Elle aboutit, au niveau de l'assiette du mangeur, à la construction d'une figure du consommateur belge qui privilégie la tendreté au détriment du goût, et qui chasse le gras en s'appuyant sur les recommandations diététiques en faveur des viandes maigres. En termes de marché, le caractère partagé de ce cadre se lit dans le monopole de fait qu'exerce le BlancBleu Belge sur le marché belge $(98 \%$ de la viande de découpe).

La norme du maigre et du tendre constitue un cadre général autour duquel connaissances et critères de performances vont se distribuer. Ainsi, au niveau de la production, l'alliance entre le maigre et le tendre a ouvert à l'époque de nouvelles voies à l'engraissement. Là où la tendreté était autrefois obtenue après abattage par maturation des carcasses (grasses), le nouveau cadre du maigre et du tendre impose, grâce à un engraissement précoce, un attendrissement avant abattage que l'on pourrait requalifier $\mathrm{d}^{\prime}$ «attendrissement sur pattes». Cela a supposé la production de nouvelles connaissances qui se sont traduites dans des références techniques (courbe de gain quotidien moyen précoce) et par des pratiques telles que la limitation de la période de croissance (volume carcasse) et la superposition de la période

\footnotetext{
${ }^{4} \mathrm{Au}$ sein de la race Blanc-Bleu Belge, il existe, aux côtés du modèle dominant culard, race hyperviandeuse, un modèle marginal, le rameau «Blanc-Bleu Belge mixte », dont la finalité est de préserver le caractère initialement mixte (lait et viande) de la production.
}

d'engraissement (développement musculaire) et d'une finition précoce (dépôt gras intramusculaire). La transformation la plus radicale, qui va rendre fortement irréversibles les choix décrits, se joue au niveau de la sélection : celle-ci, en isolant et en reproduisant le gène culard, entraîne des modifications physiologiques et morphologiques dont les conséquences normatives sont cruciales pour la stabilité du référentiel. D'une part, cette sélection fait perdre au Blanc-Bleu Belge une partie de ses qualités de ruminant herbivore au profit d'un meilleur taux de conversion d'aliments concentrés : l'engraissement intensif à base de concentré devient la norme. D'autre part, les bassins hypermusclés des mères et les veaux trop conformés rendent la naissance par voie naturelle problématique. D'occasionnelle, la césarienne devient systématique, car, chemin faisant, la sélection exerce une pression négative sur la capacité de la race à mettre bas par voie naturelle. Le référentiel s'autoréférence. On voit donc comment normes et connaissances interagissent au niveau d'un maillon de la filière.

Mais comment se joue ensuite l'articulation entre maillons? C'est par la mise en place de critères de performance. Prenons le rapport entre production et transformation. Savoir-faire en matière de césarienne et engraissement, chez les éleveurs, et en matière de découpe anatomique, chez les bouchers, vont produire, à travers la requalification des systèmes de classement EUROP pour les carcasses et du tableau de découpe pour la boucherie, un nouveau critère de performance qui maximalise la valorisation de l'hypermusclé : une nouvelle catégorie de carcasses, la catégorie $S$, ou « supercularde», vient prendre la tête du classement (devenu SEUROP) et disqualifie de ce fait les autres races, qui n'atteignent dans les meilleurs cas que le niveau E. Mais, derrière ces performances $S$, qui maximalisent les rendements de carcasses et de découpe (Hanset, 1996), que sait-on des coûts cachés d'une telle opération?

S'il est ainsi partagé et articulé, le référentiel du maigre et du tendre se caractérise aussi par son côté implicite, d'autant plus puissant qu'il est souterrain. Il agit en rendant visibles certains éléments et en en effaçant d'autres parce que trop évidents ou ignorés. Ainsi, la césarienne, qui est du domaine de l'évidence pour les éleveurs, mais reste largement ignorée des consommateurs, demeure-t-elle peu questionnable. Si la question est posée de façon confidentielle, elle est réduite au registre du bien-être animal alors que, fondamentalement, le côté systématique de la césarienne soulève le problème des limites des interventions techniques en production animale (Lips et al., 2001) et, de façon plus générale, des limites d'une trajectoire de plus en plus artificialisante de l'élevage. Les recherches actuelles menées en génomique pour produire un veau dont le gène culard ne s'exprimerait qu'après la naissance (Georges, 2004) s'inscrivent dans cette même logique. Ces opacités, 
qui témoignent de la difficulté du référentiel à rendre compte du réel, montrent combien il est devenu peu discutable, rendant de moins en moins réversible le choix technologique de son projet artificialisant. Mais la force $\mathrm{du}$ référentiel du maigre et $\mathrm{du}$ tendre s'explique-t-elle uniquement par la cohérence des normes et des connaissances développées?

Les différents maillons de la filière, que sont l'élevageengraissement, l'abattage-découpe, la distribution et la consommation, ne pourraient tenir ensemble, s'entretenir et s'autoréférencer sans la force ni la cohérence des images que produit ce référentiel. Ces images sont des raccourcis qui réduisent la complexité de chacun de ces maillons et les articulent entre eux, des éleveurs aux consommateurs : Blanc-Bleu Belge dont les «culs de poulain » champions s'exposent à l'occasion des concours et que la presse professionnelle relaie sans relâche; carcasses pendues aux crochets des chambres frigorifiques dont l'alignement régulier d'arrières galbés et de flancs maigres symbolise la performance industrielle; mise en barquette apurée d'une viande dont la couleur homogène plutôt pâle, sans tâche de graisse ni aponévrose, répond aux standards diététiques; enfin, dans sa version actualisée, le steak frites d'antan revisité à la chaleur du barbecue qui fait l'apologie de la cuisson instantanée, préférée au mijotage des viandes plus grasses et plus dures. Par leur capacité à circuler, ces images sont des supports sur lesquels s'appuient les critères de performance qui articulent, comme on l'a vu, deux maillons. Ainsi, entre production et transformation, la grille de classement SEUROP des carcasses s'appuie sur l'alignement de carcasses que l'éleveur garde en tête pour se coordonner avec le boucher. De même, le tableau de découpe affiché à l'entrée de la boucherie s'appuie sur la présentation irréprochable du maigre et du tendre au client.

Aux côtés des normes et critères de performance, ces images jouent donc un rôle majeur comme ciment et stabilisateur du référentiel. Mais, par ailleurs, parce qu'elles sont réductrices, elles contribuent au côté implicite du référentiel, par leurs effets d'écran qui masquent la complexité et les conséquences de certains choix faits dans d'autres maillons de la chaîne.

Comment le référentiel bio peut-il alors se construire face à ce référentiel dont nous avons montré, à travers l'équipement en normes, connaissances et images, le caractère partagé, implicite et articulé?

\section{Référentiel bio : sous-équipement}

Une remarque préalable s'impose avant de s'interroger sur l'existence, au sein d'une filière bio, d'un cadre normatif partagé susceptible d'orienter l'action de la filière : l'agriculture biologique, comme d'autres modes de production, repose sur des savoirs riches et diversifiés, mais peu codifiés. Nous ne remettons pas en cause ces savoirs, mais nous nous interrogeons sur leur capacité à faire référence dans une logique de filière.

L'allongement des filières a induit une codification de type réglementaire. Au-delà de l'affirmation générale d'un projet de production naturelle ${ }^{5}$, ce cadrage réglementaire, partagé, se construit, comme le précise l'attendu du premier règlement européen sur l'agriculture biologique, d'abord en référence au secteur conventionnel : pas de pesticides ni d'intrants chimiques ${ }^{6}$. Dans ses principes généraux, le règlement européen sur la production animale précise, par la notion d'équilibre, le projet de production naturelle ${ }^{7}$. La réglementation belge, par l'interdiction de la césarienne systématique, proscrit alors certaines pratiques artificialisantes auxquelles est lié, comme nous l'avons montré, le référentiel du maigre et du tendre ${ }^{8}$. En particulier, l'exclusion réglementaire de la césarienne doit-elle entraîner une adaptation du cadre du Blanc-Bleu Belge qui viserait à limiter le caractère culard ou, au contraire, des transformations plus profondes qui porteraient à la fois sur les normes et critères de performance (le troupeau plutôt que l'individu) et sur les connaissances? Le changement de race ne questionne-til pas les normes et les connaissances distribuées dans la filière?

Cette question se posait pour le groupement d'une vingtaine d'éleveurs bovins bio engagés dans une démarche de filière avec un grand distributeur. Leur stratégie adaptative visait à s'ajuster au critère de performance exigé par le transformateur et le distributeur, à savoir l'idéal des carcasses de type $\mathrm{S}$. Le choix des races Blonde d'Aquitaine ou Limousine était alors à la fois cohérent avec l'équipement des producteurs et aligné sur les exigences du maigre et du tendre portées par les transformateurs, les distributeurs et les consommateurs.

\footnotetext{
5 «Les principaux buts de l'agriculture biologique sont [...] un travail compatible avec les cycles naturels et les systèmes vivants à travers le sol, les plantes et les animaux au sein d'un système de production complet, une reconnaissance du large impact social et écologique de et au sein de la production et des systèmes de transformation [... ] $»$ (IFOAM, 2004).

6 Règlement CEE 2091/92 du 24 juin 1991.

7 «[... ] la production animale contribue à l'équilibre des systèmes de production agricole, [...] utilise des ressources naturelles renouvelables $[\ldots]$ est une production liée au sol [...] et le nombre d'animaux par unité de surface doit être limité de façon à assurer une gestion intégrée des productions animales et végétales [.. . ]» (Règlement CE 1804/1999 du 19 juillet 1999).

8 « [...] L'utilisation des races dont les problèmes de mise bas nécessitent l'usage de la césarienne doit être évitée, la césarienne n'est autorisée que pour sauver la vie d'un animal ou pour lui éviter des souffrances. [... ] on considère que cette règle est respectée pour les troupeaux de type viandeux d'un élevage donné si 5 ans après l'entrée en conversion, le nombre de naissances naturelles est et reste supérieur à $80 \%$ des naissances de l'année [... ] » (Arrêté ministériel du 30 octobre 1998).
} 
Mais il se révèle incompatible avec le projet d'élevage naturel que défend la production animale bio, car l'obligation de pâturage et la limitation de la période d'engraissement en stabulation à 3 mois ne sont pas respectées : dans les faits, la plupart des taurillons bio Blonde $\mathrm{d}^{\prime}$ Aquitaine ou Limousine passent toute leur seconde année en stabulation.

Dans le référentiel bio, l'obligation de pâturage renvoie à la norme d'équilibre naturel qui favorise la consommation de ressources à faible impact environnemental (pâtures plutôt que concentrés), tout en respectant les besoins en espaces ouverts des animaux. Les éleveurs sont sous-équipés face à cette exigence, contraire à leurs compétences d'engraisseurs intensifs basées sur un concept de finition précoce en stabulation et à base de concentrés. Comment, en effet, valoriser le fourrage jusqu'en phase de finition, comment gérer une finition dès lors plus tardive? De plus, comment renoncer à ce qu'ils maîtrisent lorsque, engagés dans cette démarche, ils se retrouvent sous la pression de la performance conjointe $\mathrm{du}$ « $\mathrm{S}$ » et $\mathrm{du}$ « maigre et tendre » que réclament transformateurs, distributeurs et consommateurs? Comment s'en détourner, si c'est précisément la validation que l'éleveur recherche sur le bon de livraison que lui donne l'abattoir? Il est dès lors très difficile aux éleveurs de résister à l'effet de lock-in ${ }^{9}$ qu'impose le reste de la filière? Car, bien qu'ils certifient l'ensemble de la filière, les principes et les règles du référentiel bio sont avant tout tournés vers l'obligation de moyens. Ils ne portent donc pas à s'interroger sur les changements en chaîne qu'implique un autre concept d'élevage, et en particulier d'engraissement. Qu'implique, pour la transformation et la consommation, l'obligation de pâturage? En matière de transformation, d'autres choix de découpe sont pourtant possibles, qui ne sont pas sans conséquences sur les modes de production (Thrift, 2003). Mais un tel questionnement ne devrait-il pas, d'abord, passer par les consommateurs?

Or, face à une figure du consommateur « conventionnel », définie dans un registre industriel sur la base de deux critères simples (le maigre et le tendre), qui demeure cloisonnée et opaque sur les conséquences des choix en matière $\mathrm{d}^{\prime}$ artificialisation de la production, la figure du consommateur bio, initialement construite sur la délégation par la confiance au producteur (obligation de moyens), est quant à elle sous-équipée. Prenons la question du goût. Au dire de très nombreux

\footnotetext{
${ }^{9}$ Les effets lock-in, ou verrouillages technologiques, sont des mécanismes qui font que, lorsque l'on est engagé dans une voie, elle devient plus facile, autoréférente, parce qu'elle atteint un certain degré d'irréversibilité. De tels verrouillages technologiques ont eu lieu par le passé sur de grandes filières industrielles : autour du train et du charbon au XIX ${ }^{\mathrm{e}}$ siècle ; autour de la voiture, de la route et du pétrole dans les années 1960 ; autour du nucléaire et de l'électricité, en France, dans les années 1970.
}

consommateurs, le goût est un critère de performance essentiel du bio. Mais il s'agit d'un point de vue normatif en réalité très controversé : de façon générale, on ne peut tirer de conclusions gustatives en faveur du bio (Bourn et Prescott, 2002), et, de façon particulière, là où la carotte bio peut éventuellement faire la différence, la viande bio se heurte aux difficultés complexes de la qualification des produits carnés, et plus particulièrement de la viande bovine (Stassart, 2003b). Si une différence en matière de goût peut se construire, elle sera fragile, exigeant d'abord de la renforcer en la reliant à d'autres critères pertinents pour les consommateurs (pâturage, bien-être), puis de fournir à ceux-ci les compétences nécessaires pour apprécier cette différence.

La construction d'un référentiel n'est pas qu'une question de choix techniques articulant normes et connaissances : comme on l'a décrit au sujet du référentiel conventionnel, elle se nourrit et nourrit la filière d'images, qui permettent aux acteurs de se projeter et de recomposer leurs équipements normativo-cognitifs. Or, $c^{\prime}$ est sur cette question des images que le référentiel bio bovin est le plus pauvre. Si l'image de verts pâturages et du troupeau allaitant renvoie bien au principe d'équilibre naturel et d'autonomie qui sous-tend le modèle d'association élevage-polyculture cher au bio, le concept même d'élevage spécialisé viandeux et ses traductions dans des principes tels que ceux d'engraissement ou de finition et dans des expressions telles que " une bête en état», définis dans le référentiel du maigre et du tendre, ne connaissent pas de traduction dans le référentiel bio. Au-delà du «cul de poulain », que l'exclusion de la césarienne rend inaccessible dans le référentiel bio, dans quelle image l'éleveur belge, de Limousine ou d'Angus, peut-il alors se projeter? Cette image impose-t-elle une vision de la performance individuelle ou propose-t-elle un équipement à l'échelle du troupeau? Ce vide d'images qui feraient sens, ces carences, génèrent chez les éleveurs bio belges une profonde incompréhension face à l'efficacité d'autres référentiels (par exemple chez les voisins bio français) qui, à leurs yeux, frisent l'amateurisme.

Le débat sur la conventionnalisation du bio peut-il faire l'économie d'une telle approche qui met au jour une série de carences dans le référentiel de filière et révèle son sous-équipement, comparé au référentiel dominant du maigre et du tendre? De façon symétrique, cela peut stimuler la réouverture d'une série de questions sur le côté implicite de ce référentiel du maigre et du tendre. D'abord sur la trajectoire artificialisante qui le sous-tend: aujourd'hui la césarienne, demain la transgenèse? Ensuite sur le suréquipement de certaines de ses pratiques, telles que le contrôle du parasitisme - faut-il éradiquer ou accompagner les parasites? - qui, nous pourrions le démontrer, évite la question du rapport au vivant au lieu de l'explorer. Enfin, cela n'exclut pas, sur certains aspects, un sous-équipement peu questionnable, tel que 
celui des pratiques des consommateurs, dont le résultat est de rendre le mangeur belge de moins en moins compétent face à d'autres alternatives. La situation actuelle de non-débat sur ce que certains considèrent comme des anomalies du maigre et du tendre montre combien ce cadre de référence est devenu d'autant plus irréversible qu'il s'est suréquipé en termes de normes, de critères, de connaissances et d'images, et réifié par les acteurs de la filière, à qui il permet de comprendre le réel en limitant sa complexité (Muller, 1990). Face à ce suréquipement global du " maigre et tendre », la traduction nationale du référentiel bio est bien sous-équipée. Transformer les seules normes de production au travers des réglementations ne suffit donc pas à transformer l'ensemble d'un référentiel, auquel les différents maillons de la filière restent très attachés par un mécanisme de lock-in que développe le suréquipement (Guthman, 2004). Face à notre question de départ, qui était celle de l'interprétation des transformations observées dans l'allongement des filières bio, notre argument est donc qu'un référentiel de filière, pour être durable, doit être équipé d'un ensemble de normes, de connaissances et d'images qui font système ${ }^{10}$.

De surcroît, bien que construites dans l'interaction entre acteurs, ces matrices normativo-cognitives tendent à s'autonomiser par rapport à leur processus de construction et à s'imposer aux acteurs comme modèle dominant d'interprétation du monde (Muller, 2000). C'est leur caractère indiscutable et non dit qui produit ce que nous appelons des effets d'opacité, comme par exemple pour la césarienne ou pour la norme $\mathrm{du}$ « tendre » qui, au niveau du produit, empêche le consommateur de développer une compétence en matière de goût.

\section{Conclusion}

Sans nous laisser enfermer dans le jeu courant des acteurs sociaux du modèle conventionnel (Godard et Hubert, 2002), nous faisons l'hypothèse que la question de l'équipement de la transformation d'un référentiel a une portée bien plus large que celle de notre cas. Des observations dans le secteur bio de la pomme de terre donnent à penser que le modèle de la Bintje y joue le même rôle de référence que le Blanc-Bleu Belge, empêchant notamment l'émergence d'un nouveau référentiel qui s'appuierait sur des variétés plus rustiques. De même, mais à une échelle plus globale, on peut s'interroger sur les effets d'opacité du référentiel global laitier, qui s'est imposé notamment à travers l'hégémonie du modèle Holstein, et ses dimensions implicites dont il est dit peu de choses en termes de production (Landais, 1996; Micoud, 2003) et dont d'autres conséquences, tel le lien

\footnotetext{
10 Un référentiel repose également sur une attribution précise des rôles qui resterait à expliciter : naisseur, éleveur, engraisseur...
}

possible avec la croissance des allergies au lait chez les jeunes enfants, restent à analyser (Riedler et al., 2001).

Plus largement, le cas très spécifique du secteur de la viande en Belgique illustre la difficulté qu'il y a à construire des filières de production-consommation plus durables. Dans les cas où, comme ici, les logiques adaptatives semblent vouées à l'échec du fait des fortes irréversibilités cachées des filières dominantes, il y a un vaste chantier d'innovations coordonnées dans lequel sciences de la nature, recherches techniques et action collective devraient s'impliquer. Encore faudra-t-il y associer les acteurs dans une démarche d'apprentissage mutuel.

\section{Remerciements}

Nous remercions Claire Lamine et Marc Mormont pour leurs commentaires et leurs encouragements, l'équipe des chercheurs du SEED (Socio-économie Environnement Développement, de la section Systèmes agraires du CRA-W (Centre wallon de recherches agronomiques), ainsi que des collègues français du département des Sciences pour l'action et le développement de l'Inra qui ont nourri notre réflexion. Enfin, le volet empirique de ce travail a été rendu possible grâce à l'étroite collaboration d'éleveurs et d'un distributeur belges engagés dans la démarche bio.

\section{Références}

Bonneval, L. de, 1993. Systèmes agraires, systèmes de production : vocabulaire français-anglais avec index anglais, Paris, INRA Éditions.

Bourn, D., Prescott, J., 2002. Comparaison entre la valeur nutritive, les qualités sensorielles et la salubrité des aliments produits de manière biologique et conventionnelle. Compte rendu critique, Food Science and Nutrition, 42, 1-34.

Buck, D., Getz, C., Guthman, J., 1997. From Farm to Table: The Organic Vegetable Commodity Chain of Northern California, Sociologia Ruralis, 37, 1, 3-20.

Callon, M., 1986. Éléments pour une sociologie de la traduction. La domestication des coquilles Saint-Jacques et des marins pêcheurs dans la baie de Saint-Brieuc, L'Année sociologique, 36, 169-208.

Coombes, B., Campbell, H., 1998. Dependent Reproduction of Alternative Modes of Agriculture: Organic Farming in New Zealand, Sociologia Ruralis, 38, 2, 127-145.

Georges, M., 2004. La sélection animale à l'ère de la génomique, Recherche en agriculture wallonne Réseau d'excellence, Namur, Direction générale de l'Agriculture.

Godard, O., Hubert, B., 2002. Rapport sur le développement durable, Paris, INRA.

Goodman, D., Sorj, B., Wilkinson, J., 1987. From Farming to Biotechnology, Oxford, Basil Blackwell.

Guthman, J., 2004. The Trouble with Organic Lite in California: A Rejoinder to the 'Conventionalisation' Debate, Sociologia Ruralis, 44, 3, 301-316. 
Hall, A., Mogyorody, V., 2001. Organic Farmers in Ontario: An Examination of the Conventionalization Argument, Sociologia Ruralis, 41, 4, 399-422.

Hanset, R., 1996. Le Blanc-Bleu Belge face à la nouvelle donne économique, Les Élevages belges, 3, 12-21.

Hubert, B., 2002. Le traitement du long terme et de la prospective dans les zones ateliers (suite). Les rapports entre chercheurs et acteurs, Natures Sciences Sociétés, 10, 4, $51-62$.

IFOAM, 2004. Principles of Organic Agriculture, consulté le 15 octobre 2004 sur le site Internet http://redaktion.ifoam.org/intranet/about_ifoam/principles/ index.html

Jobert, B., Muller, P., 1987. L'État en action : politique publique et corporatismes, Paris, PUF.

Lampkin, N., Padel, S., 1994. The Economics of Organic Farming, Wallingford, CAB International.

Landais, É., 1996. Élevage bovin et développement durable, Le Courrier de l'environnement de l'INRA, 29, 59-70.

Lips, D., De Tavernier, J., Decuypere, E., Van Outryve, J., 2001. Ethical Objections to Cesareans: Implication on the Future of the Belgian White Blue, in Matias Pasquali (Ed.), EurSafe 2001, Food Safety, Food Quality, Food Ethics, The Third Congress of the European Society for Agricultural and Food Ethics, 3-5 October, Florence, Italy, Preprints, 291-294.
Micoud, A., 2003. Ces bonnes vaches aux yeux si doux, in Dubost, F., Lizet, B. (Eds), Bienfaisante nature, Paris, Le Seuil, 217-236.

Muller, P., 1990. Les Politiques publiques, Paris, PUF.

Muller, P., 2000. L'analyse cognitive des politiques publiques: vers une sociologie politique de l'action publique, Revue française de science politique, 50, 2, 189-209.

Riedler, J., Braun-Fahrlander, C., et al., 2001. Exposure to farming in early life and development of asthma and allergy: a cross-sectional survey, The Lancet, 358, 1129-1133.

Smith, E., Marsden, T. 2004. Exploring the limits to growth in UK organics: beyond the statistical image, Journal of Rural Studies, 20, 3, 345-357.

Stassart, P., 2003a. Hybridation. Comment un collectif en rencontre un autre, in Stassart, P., Produits fermiers : entre qualification et identité, Bruxelles, PIE-Peter Lang, 149-215.

Stassart, P., 2003b. Metabolising risk: food scares and the un/remaking of Belgian beef, Environment and Planning A, 35, 449-462.

Stassart, P. Mormont, M., Jamar, D., Stilmant, D. Aertsens, J., Van Huylenbroeck, G., à paraître. Comment l'agriculture biologique contribute-t-elle aux modes de production et de consommation durable? Rapport final, Arlon.

Thrift, N., 2003. Qualification de l'origine des viandes bovines selon les manières de produire. Thèse en sciences zootechniques, Département de Sciences animales, INA P-G, Paris. 